\title{
LEARNING CONTROL OF ROBOT MANIPULATORS IN TASK SPACE
}

\author{
K. M. Dogan, E. Tatlicioglu, (1) E. Zergeroglu, and K. Cetin
}

\begin{abstract}
Two important properties of industrial tasks performed by robot manipulators, namely, periodicity (i.e., repetitive nature) of the task and the need for the task to be performed by the end-effector, motivated this work. Not being able to utilize the robot manipulator dynamics due to uncertainties complicated the control design. In a seemingly novel departure from the existing works in the literature, the tracking problem is formulated in the task space and the control input torque is aimed to decrease the task space tracking error directly without making use of inverse kinematics at the position level. A repetitive learning controller is designed which "learns" the overall uncertainties in the robot manipulator dynamics. The stability of the closed-loop system and asymptotic end-effector tracking of a periodic desired trajectory are guaranteed via Lyapunov based analysis methods. Experiments performed on an in-house developed robot manipulator are presented to illustrate the performance and viability of the proposed controller.
\end{abstract}

Key Words: Learning control, task space control, robot manipulators.

\section{INTRODUCTION}

For control systems having nonlinear components in their dynamics, including robot manipulators, various control schemes are studied in the literature [1-5]. Among these schemes, feedback linearization or computed torque methods requires the exact knowledge of the model of the nonlinear system. Since exact knowledge of the system model is generally unavailable, this method seems impractical. When the system model has structured/parametric uncertainties, adaptive control techniques can be utilized [6,7]. While dealing with structured uncertainties successfully, adaptive methods fail to deal with unstructured uncertainties. To deal with unstructured uncertainties, robust control techniques can be utilized $[8,9]$. But these methods require either discontinuous feedback (i.e., variable structure or sliding mode controllers) or high gain feedback. A class of robust controllers that does not require neither discontinuous feedback nor high control gains is the learning controllers [10-13]. Learning controllers are classified as robust controllers in the sense that they do not require exact knowledge of system dynamics. Similar to the adaptive controllers, learning controllers also include an

Manuscript received May 30, 2016; revised June 12, 2017; accepted July 30, 2017.

The authors are with the Department of Electrical \& Electronics Engineering, Izmir Institute of Technology, 35430, Urla, Izmir, Turkey.

E. Tatlicioglu is the corresponding author, (e-mail: enver@iyte.edu.tr).

E. Zergeroglu is with the Department of Computer Engineering, Gebze Technical University, 41400, Gebze, Kocaeli, Turkey (Email: e.zerger@gtu.edu.tr).

This research was funded by The Scientific and Technological Research Council of Turkey via grant number $113 \mathrm{E} 147$. update law. Different from the adaptive controllers, learning controllers aim to regulate or overcome uncertainties without the knowledge of parametric uncertainties.

Most industrial robot applications require tasks to be performed in a repetitive manner. Considering nonlinear robot dynamics and existence of several uncertainties, use of model based controllers seems imperative to increase the tracking performance. Learning controllers loom large among the class of model based controllers due to the periodic nature of the desired tasks and also their capabilities to deal with time-varying uncertainties without requiring of high gain/frequency feedback components.

The research on learning controllers was initiated by [10]. Several extensions were proposed in [11-16] where the main focus was the design of different update rules for the learning component to increase robustness. In [12] and [13], repetitive learning controllers were designed with the use of kernel and unknown influence functions in their update laws. In [10] and [11], after assuming a restrictive assumption that the robot manipulator returning to the same initial position after each iteration, betterment learning algorithms were proposed. In [17], the robustness of these controllers were investigated. Some line of research has focused on utilizing adaptive components with the learning controllers to compensate for parametric uncertainties. In [15], asymptotic convergence of tracking error was proven via designing an adaptive iterative learning controller. In [14], the design of an adaptive learning controller fused with a saturation function based feed-forwar learning component was presented where asymptotic stability of the closed-loop system was proven via Lyapunov based methods. 
Via identifying the Fourier coefficients of the input reference signal, an adaptive learning proportional derivative type controller was presented in [16]. In [18], a sliding mode based repetitive learning controller was designed for tracking control of robot manipulators subject to actuator saturation. Recently, in [19-23], Verrelli et al. researched several aspects of a linear repetitive learning control method where Padé approximation was made use of.

Several problems were addressed via the design of learning controllers and their extensions. However, almost all of the learning controllers designed for robot manipulators were joint space controllers. That is; main control objectives were to track periodic joint positions as opposed to tracking a periodic end-effector position. Only a few past works addressed designing iterative learning controllers where the desired trajectory is specified in task space $[24,25]$. For both designs, the convergence of the task space tracking errors to the origin were guaranteed. However, requiring the calculation of inverse kinematics on position level was the main shortcoming of the proposed controllers. Specifically, solving for the inverse kinematics problem typically involves solving a nonlinear system of equations with trigonometric functions. Issues such as singularity, multiple (non-unique) solutions (as in the case of 'elbow up' and 'elbow down' configurations for a robot arm), and no solution (as in the case in which the specified trajectory goes beyond the workspace of the mechanism) can often come up, further complicating the solution process. The complexity in the inverse kinematics problem is compounded even more for parallel link manipulators [26]. In [27], the problem of operational/task space tracking control of a robot manipulator is considered where simulation results implemented on a two link planar robot are presented to illustrate the viability of the proposed learning control scheme.

In this paper (preliminary results have appeared in [27]), design of a task space learning controller without utilizing inverse kinematic calculations on position level is aimed. The control problem is further complicated by the presence of uncertainties in the robot dynamics. A model-independent controller fused with a model-independent learning component is designed. The overall stability of the closed-loop system and the convergence of the task space tracking error are guaranteed via Lyapunov based arguments. Asymptotic end-effector tracking is ensured via learning the uncertainties associated with the robot dynamics after each period of the desired end-effector trajectory. Experiments performed on a three degree of freedom robot manipulator with three links and three actuators to illustrate the viability of the proposed task space learning controller.
In this paper, motivated by the work of [5], we present the design and associated analysis of a task-space learning controller. Unlike standard learning controllers, the key feature of the proposed architecture does not utilize, possibly complicated, manipulator inverse kinematic calculations. This is accomplished by designing an auxiliary term to the control signal. Specifically, the proposed controller formulation achieves asymptotic end-effector tracking by learning the uncertainties associated with the system dynamics after each period of the desired end-effector pose. Closed-loop stability and convergence of the error signals are guaranteed via Lyapunov based arguments and experiment results implemented on a three link planar robot are presented to illustrate the viability of the proposed learning control scheme.

\section{KINEMATIC AND DYNAMIC MODELS}

The dynamic model of an $n$ degree of freedom revolute joint robot manipulator has the following form $[2,28]$

$$
M(\theta) \ddot{\theta}+C(\theta, \dot{\theta}) \dot{\theta}+G(\theta)+F(\dot{\theta})=\tau
$$

where $\theta(t), \dot{\theta}(t), \ddot{\theta}(t) \in \mathbb{R}^{n}$ denote joint positions, velocities, and accelerations, respectively, $M(\theta) \in \mathbb{R}^{n \times n}$ is the positive-definite and symmetric inertia matrix, $C(\theta, \dot{\theta}) \in \mathbb{R}^{n \times n}$ represents the centripetal-Coriolis terms, $G(\theta) \in \mathbb{R}^{n}$ denotes the gravitational effects, $F(\dot{\theta}) \in \mathbb{R}^{n}$ is the frictional effects, and $\tau(t) \in \mathbb{R}^{n}$ is the control input torque.

Property 1. The inertia matrix satisfies the following inequalities [4]:

$$
m_{1} I_{n} \leq M(\theta) \leq m_{2} I_{n}
$$

where $m_{1}, m_{2} \in \mathbb{R}$ are known positive bounding constants, and $I_{n} \in \mathbb{R}^{n \times n}$ is the standard identity matrix.

Property 2. The inertia and centripetal-Coriolis matrices satisfy the following skew-symmetry relationship [4]

$$
\xi^{T}(\dot{M}-2 C) \xi=0 \forall \xi \in \mathbb{R}^{n} .
$$

Property 3. The centripetal-Coriolis matrix satisfies the following switching property [1]

$$
C(\xi, \nu) \eta=C(\xi, \eta) \nu \forall \xi, \nu, \eta \in \mathbb{R}^{n} .
$$

Property 4. The dynamic modeling terms in (1) can be upper bounded as [1,29]

$$
\|M(\xi)-M(v)\|_{i \infty} \leq \zeta_{M 1}\|\xi-v\|
$$




$$
\begin{aligned}
& \|C(\xi, \eta)\|_{i \infty} \leq \zeta_{C 1}\|\eta\| \\
& \|C(\xi, \eta)-C(\nu, \eta)\|_{i \infty} \leq \zeta_{C 2}\|\xi-v\|\|\eta\| \\
& \|G(\xi)-G(v)\| \leq \zeta_{G}\|\xi-v\| \\
& \|F(\xi)-F(v)\| \leq \zeta_{F}\|\xi-v\|
\end{aligned}
$$

$\forall \xi, \nu, \eta \in \mathbb{R}^{n}$, where $\zeta_{M 1}, \zeta_{C 1}, \zeta_{C 2}, \zeta_{G}, \zeta_{F} \in \mathbb{R}$ are positive bounding constants.

The task space position, denoted by $x(t) \in \mathbb{R}^{n}$, is obtained as

$$
x=f(\theta)
$$

where $f: \mathbb{R}^{n} \rightarrow \mathbb{R}^{n}$ is the forward kinematics. Differentiating (10) yields

$$
\dot{x}=J \dot{\theta}
$$

where $J(\theta) \in \mathbb{R}^{n \times n}$ is the Jacobian matrix defined as

$$
J \triangleq \frac{\partial f}{\partial \theta} .
$$

Assumption 1. Inverse kinematics at the position level, denoted by $h: \mathbb{R}^{n} \rightarrow \mathbb{R}^{n}$, has the following form [2]

$$
\theta=h(x) .
$$

Utilizing $h$ along with Mean Value Theorem in [25] allows the following to be satisfied

$$
\|h(\xi)-h(v)\| \leq \zeta_{h}\|\xi-v\| \forall \xi, v
$$

where $\zeta_{h} \in \mathbb{R}$ is a known positive bounding constant. Differentiating (13) yields

$$
\dot{\theta}=J^{-1}(x) \dot{x}
$$

where $J^{-1}(x) \in \mathbb{R}^{n \times n}$ (the notations $J^{-1}(x)$ or $J^{-1}(\theta)$ will be utilized interchangeably throughout this paper) is the inverse Jacobian matrix (also referred to as inverse kinematics at the velocity level)

$$
J^{-1}(x) \triangleq \frac{\partial h(x)}{\partial x} .
$$

Remark 1. It is emphasized that the inverse kinematics at the position level given in (13) is introduced for analysis purposes only. The design of the subsequent learning controller will be made without requiring the inverse kinematics at the position level.

Remark 2. Similar to the existing works in task space tracking control literature, all kinematic singularities are considered to be always avoided and thus $J^{-1}(\theta)$ exists $\forall \theta$ [2]. This assumption can alternatively be stated as the minimum singular value of the Jacobian matrix being greater than a small positive constant (i.e., $\left.\min \left\{\|J(\theta)\|_{i \infty}\right\}>\mu>0\right)$.

Assumption 2. The dynamic modeling terms $M(\theta)$, $C(\theta, \dot{\theta})$, and $G(\theta)$, and the kinematic terms $J(\theta)$ and $J^{-1}(\theta)$ depend on $\theta(t)$ via trigonometric functions only and thus they are bounded for all possible $\theta(t)$.

Property 5. Based on Assumption 2, following bounds can be obtained

$$
\zeta_{J 1}<\|J\|_{i \infty}<\zeta_{J 2}
$$

from which following can be obtained

$$
\frac{1}{\zeta_{J 2}}<\left\|J^{-1}\right\|_{i \infty}<\frac{1}{\zeta_{J 1}}
$$

with $\zeta_{J 1}, \zeta_{J 2} \in \mathbb{R}$ being known positive bounding constants. The inverse Jacobian matrix satisfies

$$
\left\|J^{-1}(x)-J^{-1}\left(x_{d}\right)\right\|_{i \infty} \leq \zeta_{J 3}\left\|x-x_{d}\right\|
$$

where $\zeta_{J 3} \in \mathbb{R}$ is a positive bounding constant. When obtaining upper bounds, with an abuse of notation, only to exactly demonstrate dependence of its arguments, following notation will be used $\dot{J}^{-1}(x, \dot{x})=\frac{d}{d t}\left\{J^{-1}(x)\right\}$. The time derivative of the inverse Jacobian satisfies following bounds [4]

$$
\begin{aligned}
& \left\|\dot{J}^{-1}(\xi, \dot{\xi})\right\|_{i \infty} \leq \zeta_{J 4}\|\dot{\xi}\| \\
& \left\|\dot{J}^{-1}(\xi, \eta)-\dot{J}^{-1}(\nu, \eta)\right\|_{i \infty} \leq \zeta_{J 5}\|\xi-v\|\|\eta\| \\
& \left\|\dot{J}^{-1}(\xi, \eta)-\dot{J}^{-1}(\xi, \nu)\right\|_{i \infty} \leq \zeta_{J 6}\|\eta-v\|
\end{aligned}
$$

$\forall \xi, v, \eta \in \mathbb{R}^{n}$ where $\zeta_{J 4}, \zeta_{J 5}, \zeta_{J 6} \in \mathbb{R}$ are known positive bounding constants.

\section{LEARNING CONTROL DESIGN}

The main control objective is to design a controller that ensures tracking of a periodic desired task space trajectory under the restriction that the dynamic model being uncertain.

To quantify the tracking control objective, task space tracking error, denoted by $e(t) \in \mathbb{R}^{n}$, is defined as

$$
e \triangleq x_{d}-x
$$

where $x_{d}(t) \in \mathbb{R}^{n}$ is the periodic desired task space trajectory. The desired task space trajectory along with its 
first two time derivatives satisfy $x_{d}(t)=x_{d}(t-T), \dot{x}_{d}(t)=$ $\dot{x}_{d}(t-T), \ddot{x}_{d}(t)=\ddot{x}_{d}(t-T)$ with $T$ being the known positive period, and $\left\|x_{d}(t)\right\| \leq \zeta_{x_{d}},\left\|\dot{x}_{d}(t)\right\| \leq \zeta_{\dot{x}_{d}},\left\|\ddot{x}_{d}(t)\right\| \leq$ $\zeta_{\ddot{x}_{d}}$ where $\zeta_{x_{d}}, \zeta_{\dot{x}_{d}}, \zeta_{\ddot{x}_{d}} \in \mathbb{R}$ being known positive bounding constants.

Differentiating (23) via utilizing (11) yields

$$
\dot{e}=\dot{x}_{d}-J \dot{\theta} .
$$

An auxiliary error vector, denoted by $r(t) \in \mathbb{R}^{n}$, is introduced as

$$
r \triangleq J^{-1}\left(\dot{x}_{d}+\alpha e\right)-\dot{\theta}
$$

where $\alpha \in \mathbb{R}^{n \times n}$ is a constant, positive definite, diagonal gain matrix. Substituting (25) into (24) yields

$$
\dot{e}=-\alpha e+J r .
$$

First differentiating (25), then premultiplying with $M(\theta)$, and then using (1) and (25), following is obtained

$$
\begin{aligned}
M \dot{r}= & M \frac{d}{d t}\left[J^{-1}\left(\dot{x}_{d}+\alpha e\right)\right]+C J^{-1}\left(\dot{x}_{d}+\alpha e\right) \\
& -C r+G+F-\tau .
\end{aligned}
$$

An auxiliary vector, denoted by $N\left(x, \dot{x}, x_{d}, \dot{x}_{d}, \ddot{x}_{d}\right) \in \mathbb{R}^{n}$, is defined as

$$
\begin{aligned}
N \triangleq & M \frac{d}{d t}\left[J^{-1}\left(\dot{x}_{d}+\alpha e\right)\right] \\
& +C J^{-1}\left(\dot{x}_{d}+\alpha e\right)+G+F
\end{aligned}
$$

by using which, the right hand side of (27) is rewritten as

$$
M \dot{r}=-C r+N-\tau .
$$

Another auxiliary vector, denoted by $N_{d}\left(x_{d}, \dot{x}_{d}, \ddot{x}_{d}\right) \in$ $\mathbb{R}^{n}$, is defined as

$$
\begin{aligned}
N_{d} & \left.\triangleq N\right|_{x=x_{d}, \dot{x}=\dot{x}_{d}} \\
& =M\left(h\left(x_{d}\right)\right) \frac{d}{d t}\left\{J^{-1}\left(x_{d}\right) \dot{x}_{d}\right\} \\
& +C\left(h\left(x_{d}\right), J^{-1}\left(x_{d}\right) \dot{x}_{d}\right) J^{-1}\left(x_{d}\right) \dot{x}_{d} \\
& +G\left(h\left(x_{d}\right)\right)+F\left(J^{-1}\left(x_{d}\right) \dot{x}_{d}\right) .
\end{aligned}
$$

Remark 3. From (31), it is easy to see that $N_{d}$ is a function of desired task space trajectory and its time derivatives only. Since the desired task space trajectory and its time derivatives are periodic, then $N_{d}(t)$ is periodic in the sense that

$$
N_{d}(t)=N_{d}(t-T) .
$$

Furthermore, since the desired task space trajectory and its time derivatives are bounded functions of time, then
$N_{d}(t)$ and its entries can be proven to be bounded as $\left|N_{d, i}(t)\right| \leq \beta_{i} i=1, \ldots, n$ where $\beta_{i} \in \mathbb{R}$ are known positive bounding constants.

Remark 4. The difference between auxiliary vectors $N$ and $N_{d}$, denoted by $\tilde{N}\left(x, \dot{x}, x_{d}, \dot{x}_{d}, \ddot{x}_{d}\right) \in \mathbb{R}^{n}$, is defined as

$$
\tilde{N} \triangleq N-N_{d} .
$$

The norm of $\tilde{N}$ can be bounded as [30]

$$
\|\tilde{N}\| \leq \rho(\|e\|)\|z\|
$$

where $\rho(\|e\|) \in \mathbb{R}$ is a known positive non-decreasing function of its argument, and $z(t) \triangleq\left[e^{T}, r^{T}\right]^{T} \in \mathbb{R}^{2 n}$ is the combined error.

Via utilizing (30) and (33), from (29), we obtain

$$
M \dot{r}=-C r+N_{d}+\tilde{N}-\tau .
$$

The control input torque is designed as follows. (The control input torque in (36) and (37) requires $r(t)$, and from (25), it is clear that only the inverse of the Jacobian matrix is required and inverse kinematics in position level (i.e., $h$ ) is not required.)

$$
\tau=K r+k_{n} \rho^{2}(\|e\|) r+J^{T} e+\hat{N}
$$

where $K \in \mathbb{R}^{n \times n}$ is a constant, positive-definite, diagonal control gain matrix, $k_{n} \in \mathbb{R}$ is a constant, positive control gain, $\hat{N}(t) \in \mathbb{R}^{n}$ is the feed-forward learning component is updated according to

$$
\hat{N}(t)=\operatorname{Sat}_{\beta}(\hat{N}(t-T))+k_{L} r
$$

where $k_{L} \in \mathbb{R}$ is a constant positive control gain, $\beta \triangleq$ $\left[\beta_{1} \ldots \beta_{n}\right]^{T} \in \mathbb{R}^{n}$, and $\operatorname{Sat}_{\beta}(\cdot) \in \mathbb{R}^{n}$ is the vector saturation function with its entries defined as

$$
\operatorname{sat}_{\beta_{i}}\left(\kappa_{i}\right)=\left\{\begin{array}{l}
\beta_{i} \operatorname{sgn}\left(\kappa_{i}\right),\left|\kappa_{i}\right|>\beta_{i} \\
\kappa_{i},\left|\kappa_{i}\right| \leq \beta_{i}
\end{array}\right.
$$

$\forall \kappa_{i}$ where sgn $(\cdot) \in \mathbb{R}$ denotes the sign function.

Substituting (36) into (35) yields

$$
M \dot{r}=\tilde{N}+\chi-C r-k_{n} \rho^{2} r-J^{T} e-K r
$$

where $\chi(t) \in \mathbb{R}^{n}$ is defined as

$$
\chi \triangleq N_{d}-\hat{N} \text {. }
$$

From Remark 3, it is easy to obtain

$$
N_{d}(t)=\operatorname{Sat}_{\beta}\left(N_{d}(t)\right)=\operatorname{Sat}_{\beta}\left(N_{d}(t-T)\right)
$$


where the first equality is a consequence of the boundedness of the entries of $N_{d}(t)$, while the second equality is a result of the periodicity of $N_{d}(t)$.

Utilizing (37) and (41) along with (40) results in

$$
\chi=\operatorname{Sat}_{\beta}\left(N_{d}(t-T)\right)-\operatorname{Sat}_{\beta}(\hat{N}(t-T))-k_{L} r .
$$

\section{STABILITY ANALYSIS}

Theorem. The controller in (36) with the feed-forward learning component in (37) ensures boundedness of all signals under the closed-loop operation and asymptotic tracking of a periodic desired task space trajectory in the sense that

$$
\|e(t)\| \rightarrow 0 \text { as } t \rightarrow+\infty
$$

provided that the control gains are chosen to satisfy

$$
\min \left\{\alpha_{\min }, K_{\min }+\frac{k_{L}}{2}\right\}-\frac{1}{4 k_{n}}>0
$$

where $\alpha_{\min }$ and $K_{\min }$ denote the minimum eigenvalues of $\alpha$ and $K$, respectively.

Proof. A non-negative function, denoted by $V(t) \in \mathbb{R}$, is defined as

$$
\begin{aligned}
V \triangleq & \frac{1}{2} e^{T} e+\frac{1}{2} r^{T} M r \\
& +\frac{1}{2 k_{L}} \int_{t-T}^{t}\left\|\operatorname{Sat}_{\beta}\left(N_{d}(\sigma)\right)-\operatorname{Sat}_{\beta}(\hat{N}(\sigma))\right\|^{2} d \sigma .
\end{aligned}
$$

Taking the time derivative of $V(t)$ yields

$$
\begin{aligned}
\dot{V} & =e^{T} \dot{e}+\frac{1}{2} r^{T} \dot{M} r+r^{T} M \dot{r} \\
& +\frac{1}{2 k_{L}}\left\|\operatorname{Sat}_{\beta}\left(N_{d}(t)\right)-\operatorname{Sat}_{\beta}(\hat{N}(t))\right\|^{2} \\
& -\frac{1}{2 k_{L}}\left\|\operatorname{Sat}_{\beta}\left(N_{d}(t-T)\right)-\operatorname{Sat}_{\beta}(\hat{N}(t-T))\right\|^{2}
\end{aligned}
$$

where Leibniz formula was utilized. Utilizing (26) and (39) with (46) results in

$$
\begin{aligned}
\dot{V} & =e^{T}(-\alpha e+J r)+\frac{1}{2} r^{T} \dot{M} r \\
& +r^{T}\left[\tilde{N}+\chi-C r-k_{n} \rho^{2} r-J^{T} e-K r\right] \\
& +\frac{1}{2 k_{L}}\left\|\operatorname{Sat}_{\beta}\left(N_{d}(t)\right)-\operatorname{Sat}_{\beta}(\hat{N}(t))\right\|^{2} \\
& -\frac{1}{2 k_{L}}\left\|N_{d}(t)-\hat{N}(t)+k_{L} r\right\|^{2}
\end{aligned}
$$

where (40) and (42) were utilized to obtain the last line. Utilizing (3), canceling common terms and rewriting the last line results in

$$
\begin{aligned}
\dot{V}= & -e^{T} \alpha e+r^{T} \tilde{N}-k_{n} \rho^{2} r^{T} r+r^{T} \chi-r^{T} K r \\
& +\frac{1}{2 k_{L}}\left\|\operatorname{Sat}_{\beta}\left(N_{d}(t)\right)-\operatorname{Sat}_{\beta}(\hat{N}(t))\right\|^{2} \\
& -\frac{1}{2 k_{L}}\left\|N_{d}(t)-\hat{N}(t)\right\|^{2}-\left[N_{d}(t)-\hat{N}(t)\right]^{T} r \\
& -\frac{k_{L}}{2} r^{T} r \\
= & e^{T} \alpha e-r^{T} K r-\frac{k_{L}}{2} r^{T} r+\left[r^{T} \tilde{N}-k_{n} \rho^{2} r^{T} r\right] \\
& +\frac{1}{2 k_{L}}\left\|\operatorname{Sat}_{\beta}\left(N_{d}(t)\right)-\operatorname{Sat}_{\beta}(\hat{N}(t))\right\|^{2} \\
& -\frac{1}{2 k_{L}}\left\|N_{d}(t)-\hat{N}(t)\right\|^{2}
\end{aligned}
$$

where (40) was also used. For the bracketed term in (49), following bound is obtained

$$
r^{T} \tilde{N}-k_{n} \rho^{2} r^{T} r \leq \frac{1}{4 k_{n}}\|z\|^{2} .
$$

As shown in [5] and [31], following relationship is valid

$$
\left\|N_{d}(t)-\hat{N}(t)\right\|^{2} \geq\left\|\operatorname{Sat}\left(N_{d}(t)\right)-\operatorname{Sat}(\hat{N}(t))\right\|^{2} .
$$

In view of (50) and (51), from (49), we obtain

$$
\begin{aligned}
& \dot{V} \leq-e^{T} \alpha e-r^{T} K r-\frac{k_{L}}{2} r^{T} r+\frac{1}{4 k_{n}}\|z\|^{2} \\
& \leq-\left[\min \left\{\alpha_{\min }, K_{\min }+\frac{k_{L}}{2}\right\}-\frac{1}{4 k_{n}}\right]\|z\|^{2}
\end{aligned}
$$

and provided that the gain condition in (44) is satisfied, we can obtain

$$
\dot{V} \leq-\gamma\|z\|^{2}
$$

where $\gamma \in \mathbb{R}$ is a positive constant. From (45) and (54), $V(t) \in \mathcal{L}_{\infty}$. From (45), $e(t), r(t) \in \mathcal{L}_{\infty}$. Utilizing the boundedness of $e(t)$ and $r(t)$ in view of Assumption 2 along with (26), it is easy to prove that $\dot{e}(t) \in \mathcal{L}_{\infty}$. Boundedness of $e(t), \dot{e}(t), x_{d}(t)$ and $\dot{x}_{d}(t)$ can be used along with (23) and its time derivative to ensure that $x(t), \dot{x}(t) \in \mathcal{L}_{\infty}$. Utilizing $r(t) \in \mathcal{L}_{\infty}$ and properties of the saturation function in (37), $\hat{N}(t) \in \mathcal{L}_{\infty}$. From (36), it can be proven that $\tau(t) \in \mathcal{L}_{\infty}$. Utilizing $\dot{x}(t) \in \mathcal{L}_{\infty}$ and Assumption 2, from (11), $\dot{\theta}(t) \in \mathcal{L}_{\infty}$ and thus $C(\theta, \dot{\theta}) \in \mathcal{L}_{\infty}$. From (1), $\ddot{\theta}(t) \in \mathcal{L}_{\infty}$. And utilizing the above boundedness arguments with (35), $\dot{r}(t) \in \mathcal{L}_{\infty}$ is proven. 
Integrating (54) in time from $t=0$ to $+\infty$ yields

$$
\int_{0}^{+\infty}\|z(\sigma)\|^{2} d \sigma \leq \frac{1}{\gamma}(V(0)-V(+\infty)) \leq \frac{V(0)}{\gamma}
$$

from which it is easy to see that $z(t) \in \mathcal{L}_{2}$. In view of (55) and since $z(t), \dot{z}(t) \in \mathcal{L}_{\infty}$, Barbalat's Lemma in [32] can be used to prove that $\|z(t)\| \rightarrow 0$ as $t \rightarrow+\infty$, thus $\|e(t)\| \rightarrow 0$, as stated in (43).

\section{EXPERIMENTAL STUDIES}

In order to demonstrate the performance of the proposed controller, an experimental study is conducted on a robot manipulator. The 3 degree of freedom robot manipulator in Fig. 1 has articulated structure with 3 links and 3 actuators, and works on plane. Direct drive actuators of E137576 Maxon motors with the technical features of nominal voltage of $24 \mathrm{VDC}$, torque constant of $36.4 \times 10^{-3} \mathrm{Nm} / \mathrm{A}$, speed constant of $263 \mathrm{rpm} / \mathrm{V}$, nominal speed of $5530 \mathrm{rpm}$, nominal torque of $78.2 \times 10^{-3} \mathrm{Nm}$ were used. The motors are driven by Maxon Escon 36/2 DC 4-Q servo-controller with a maximum power of 72 Watts. For absolute angular measurement, AS5045 magnetic rotary encoders with a resolution of 4096 positions per revolution based on contactless magnetic sensor technology were used. The proposed control method is implemented on the computer and run on MATLAB Simulink by using Real Time Windows Target. The control inputs are transmitted to the motor drivers with analog signals and encoder signals are received as quadrature counter inputs. The data transmission between the computer and the drivers is carried out with Humusoft MF624 data acquisition board. The experimental studies run on MATLAB Simulink with a sampling rate of $0.001 \mathrm{sec}$. In the experiments, in order to obtain a

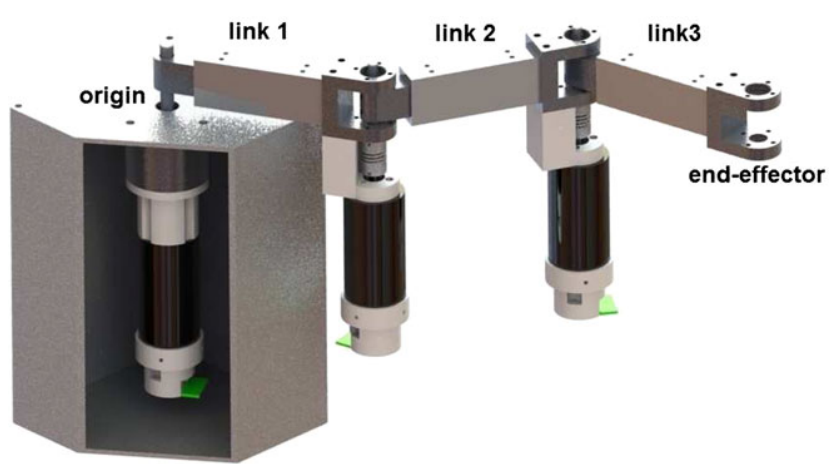

Fig. 1. 3 degree of freedom planar robot manipulator. [Color figure can be viewed at wileyonlinelibrary.com] non-redundant robot manipulator, the first link was mechanically stopped and only the last two links were utilized.

The end-effector position of the robot manipulator is obtained as

$$
x(t)=\left[\begin{array}{c}
X(t) \\
Y(t)
\end{array}\right]=\left[\begin{array}{l}
l_{1} c_{1}+l_{2} c_{12} \\
l_{1} s_{1}+l_{2} s_{12}
\end{array}\right]
$$

where the link lengths are $l_{1}=l_{2}=0.127 \mathrm{~m}$, and $s_{1}=$ $\sin \left(\theta_{1}\right), c_{1}=\cos \left(\theta_{1}\right), s_{12}=\sin \left(\theta_{1}+\theta_{2}\right), c_{12}=\cos \left(\theta_{1}+\theta_{2}\right)$. The Jacobian matrix is obtained as

$$
J=\left[\begin{array}{cc}
-l_{1} s_{1}-l_{2} s_{12} & -l_{2} s_{12} \\
l_{1} c_{1}+l_{2} c_{12} & l_{2} c_{12}
\end{array}\right] .
$$

The manipulator was initialized to be at rest at the joint position $\theta(0)=[0 \pi / 2] \mathrm{rad}$. The desired taskspace trajectory was selected as

$$
x_{d}=\left[\begin{array}{c}
0.127+0.02 \sin (0.2 t)(1-\exp (-0.1 t)) \\
0.147-0.02 \cos (0.2 t)(1-\exp (-0.1 t))
\end{array}\right][\mathrm{m}] .
$$

In the experiments, for simplicity reasons, the terms $K r+k_{n} \rho^{2}(\|e\|) r$ in the control input in (36) are considered to be combined and a constant gain is considered to be multiplying $r(t)$. Satisfactory tracking performance is obtained when the combined gain of $r(t)$ was set as $50 \times \operatorname{diag}\{1.5,1.0\}, \alpha=\operatorname{diag}\{1.5,1.0\}$ and $k_{L}=50 \times$ $\operatorname{diag}\{1.5,1.0\}$. We chose these control gains via trial and error method. However, the tuning process was relatively easy where we started with conservative (i.e., big) gains and when the experiments worked smaller control gains were tried until satisfactory tracking performance was obtained. Limits of the saturation function were selected as \pm 30 (i.e., $\beta_{1}=\beta_{2}=30$ ).

The task space tracking error $e(t)$ is shown in Fig. 2. Control input torque can be seen in Fig. 3. The desired and the actual task space trajectories can be seen from Fig. 4. From Figs 2 and 4, it is clear that the tracking objective was successfully met. Furthermore, from Fig. 2, it is observed that the proposed learning controller ensures a significant improvement on the tracking error in every period of the desired task-space trajectory which was $10 \pi \mathrm{sec}$.

To examine the results of the proposed control strategy in a comparative manner, experiments were also performed for a standard proportional integral derivative (PID) type controller of the form

$$
\tau=K_{p} e(t)+K_{i} \int_{0}^{t} e(\sigma) d \sigma+K_{d} \dot{e}(t) .
$$



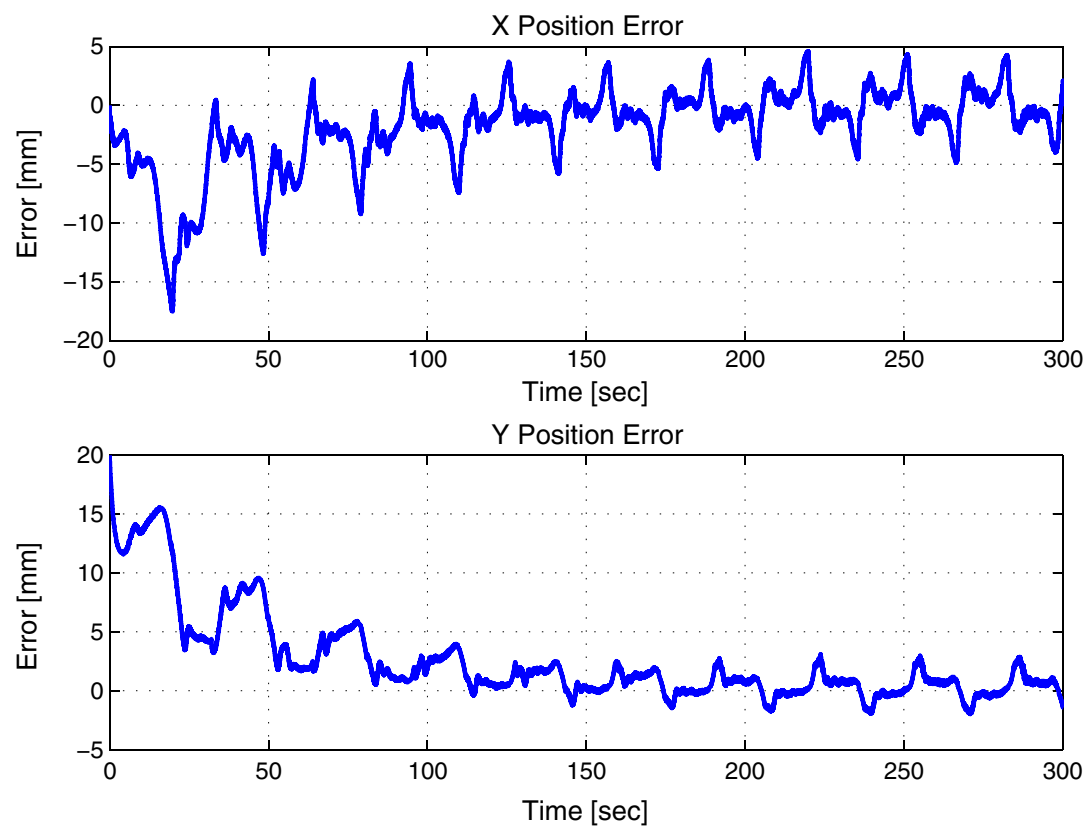

Fig. 2. Task-space position tracking error $e(t)$. [Color figure can be viewed at wileyonlinelibrary.com]
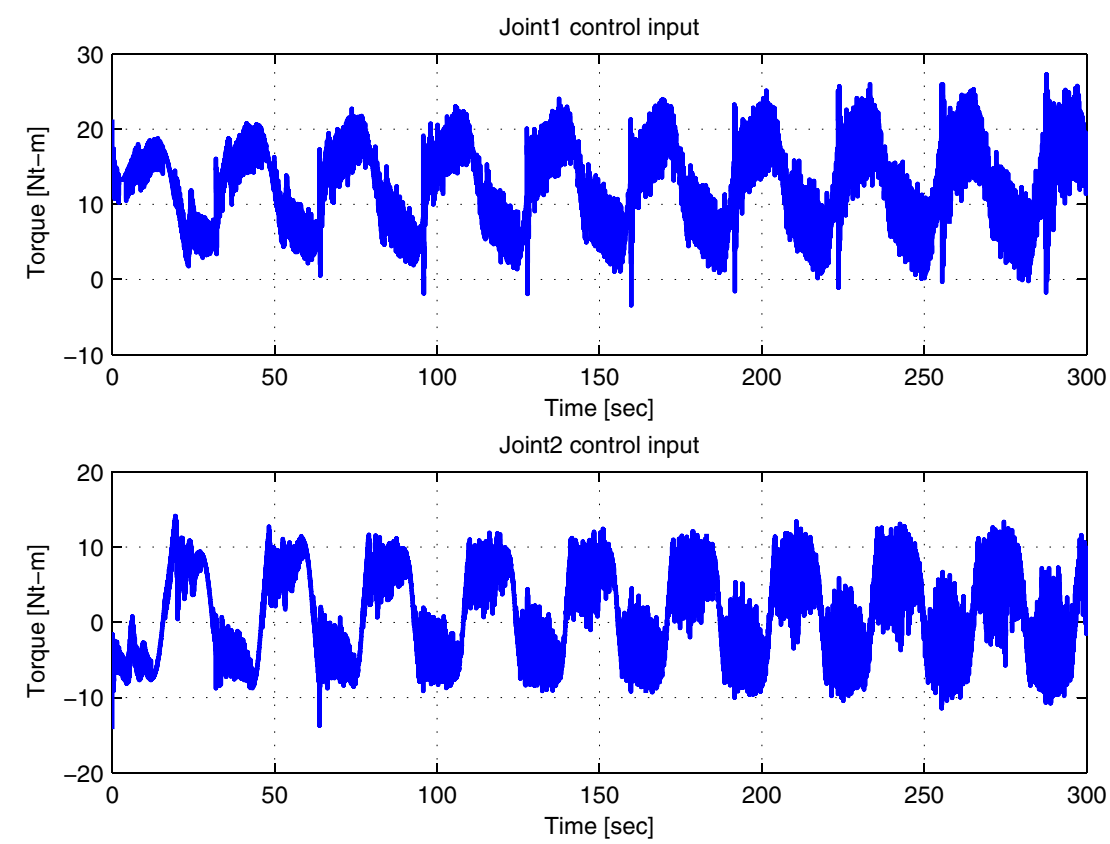

Fig. 3. Control input torque $\tau(t)$. [Color figure can be viewed at wileyonlinelibrary.com]

In (59), the controller gains were tuned via a trial and error method until both a good tracking performance and a similar performance to that of the proposed controller were achieved, and were chosen as $K_{p}=48 I_{2}$, $K_{i}=12 I_{2}, K_{d}=8 I_{2}$.
Task-space position tracking errors for both controllers are shown in Fig. 5, while in Fig. 6, control input torques are given. From Fig.5, it is clearly seen that the proposed learning controller outperforms the PID controller. Square of the integral of the norm of the 

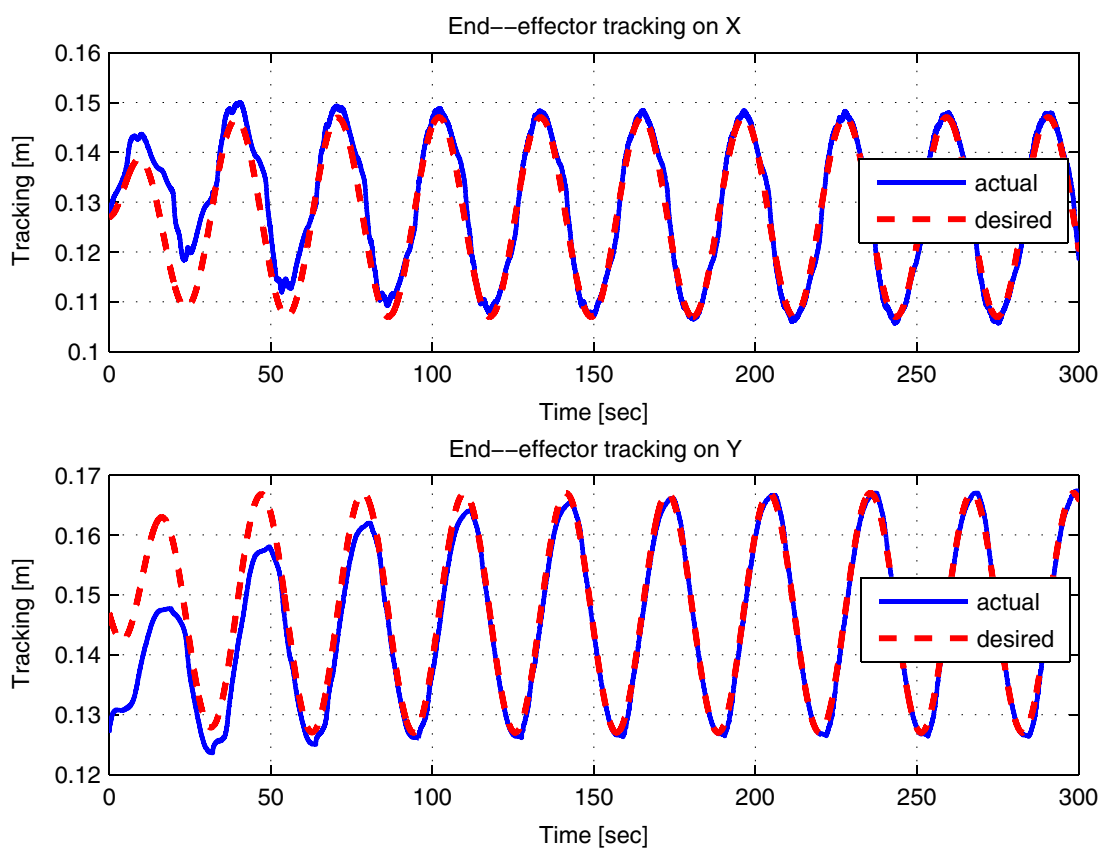

Fig. 4. Desired $x_{d}(t)$ and actual $x(t)$ task-space trajectories. [Color figure can be viewed at wileyonlinelibrary.com]
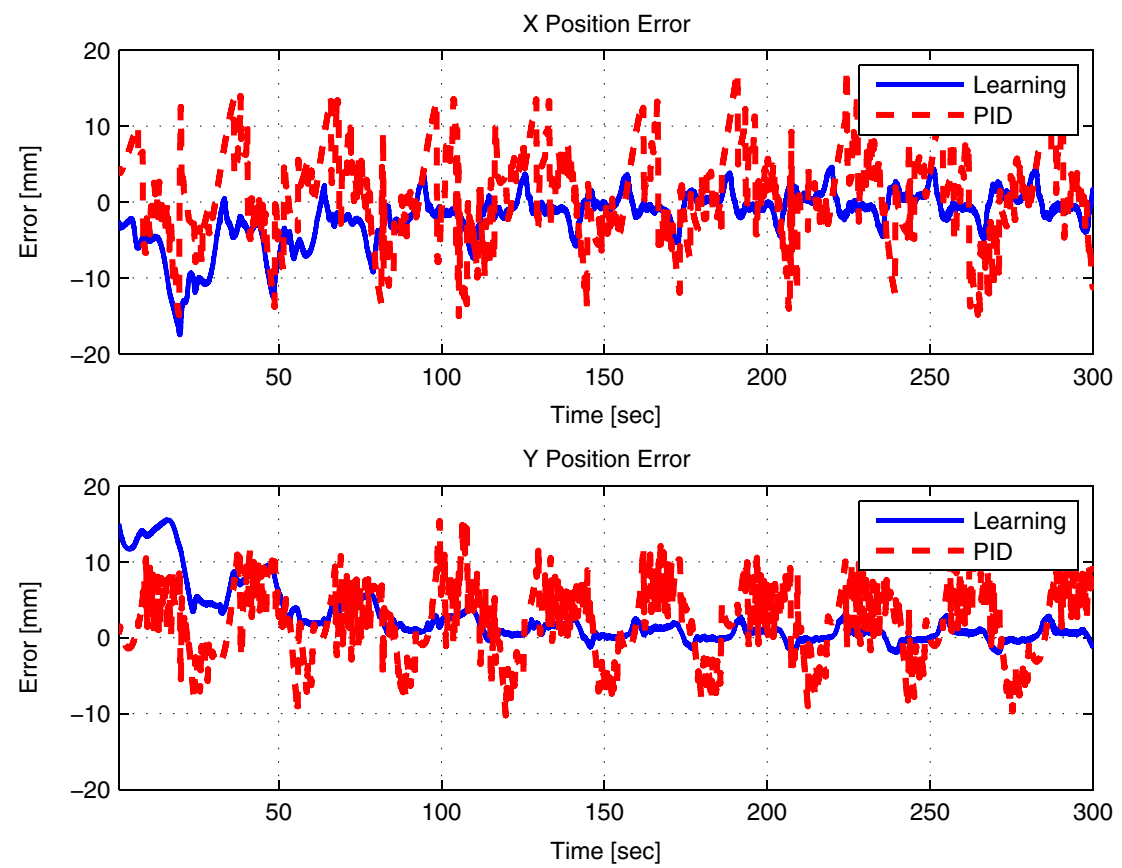

Fig. 5. Task-space position tracking errors. [Color figure can be viewed at wileyonlinelibrary.com]

task-space tracking errors (i.e., $\int_{0}^{t_{\text {final }}}\|e(t)\|^{2} d t$ ) and control input torques (i.e., $\int_{0}^{t_{\text {final }}}\|\tau(t)\|^{2} d t$ ) were calculated and recorded as performance measures during the experiments. In Table I performance measures are presented.
According to Table I, it can be said that higher control effort was needed for PID controller to obtain a close task-space tracking performance. This difference can also be seen from Fig. 6 where control input torques for both controllers are presented. 

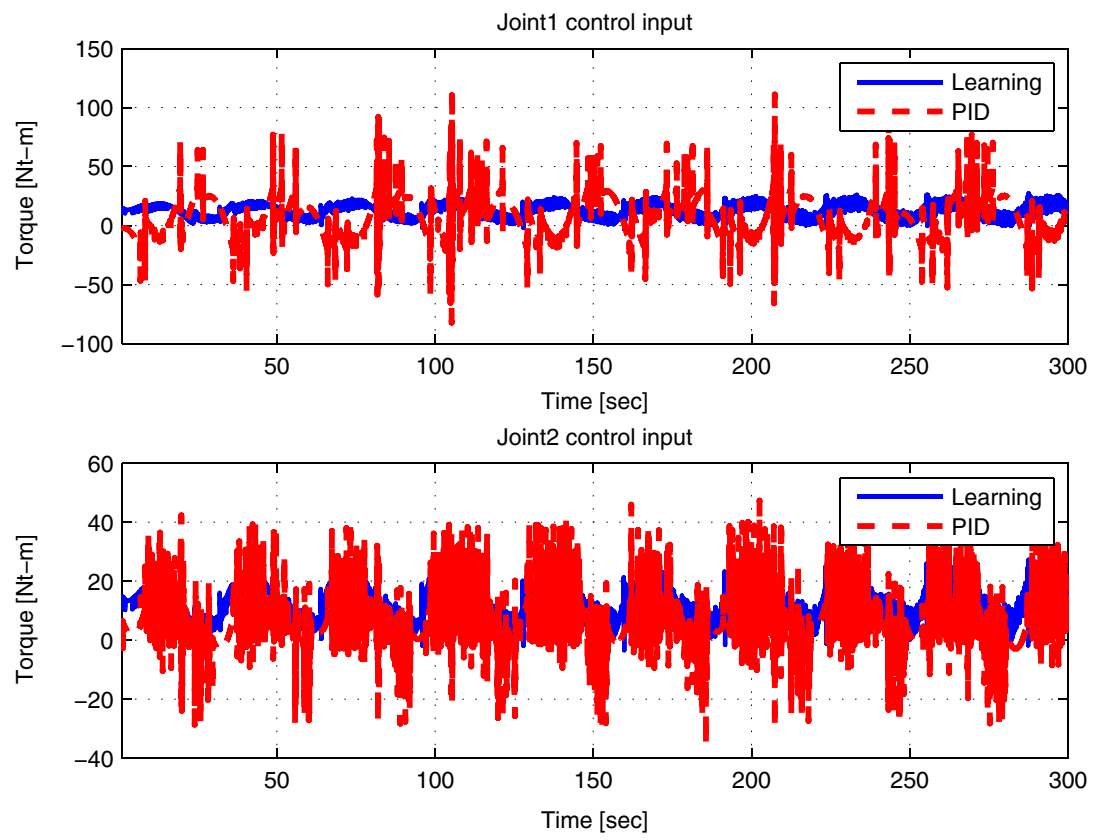

Fig. 6. Control input torques. [Color figure can be viewed at wileyonlinelibrary.com]

Table I. Performance measures

\begin{tabular}{ccc}
\hline & Learning & PID \\
\hline $\int_{0}^{t_{\text {final }}}\|e(t)\|^{2} d t$ & $1.0731 \times 10^{4}$ & $1.960 \times 10^{4}$ \\
$\int_{0}^{t_{\text {final }}}\|\tau(t)\|^{2} d t$ & $6.5118 \times 10^{4}$ & $10.696 \times 10^{4}$ \\
\hline
\end{tabular}

Remark 5. As can be observed from the control torque input graphs, after each period of the desired trajectory the control effect (the torque inputs to the system) grows. We would like to note that this is a typical behavior of learning type controllers as in each period the learning term in the controller greps more information from the overall system (i.e., learns the system) and utilizes this information to the controller. This growing behavior diminishes when the so called learning is completely done or when the saturation term in the learning component is reached.

\section{CONCLUSIONS}

The main aim of this work was to ensure end-effector tracking of a periodic desired task space trajectory. The control problem was further complicated by the dynamics of the robot manipulator being uncertain. To address all of these, in this study, we presented a novel repetitive learning controller for robot manipulators for tracking periodic task space trajectories without making use of the inverse kinematic calculations in the position level. The stability of the Closed-loop system was ensured via Lyapunov based techniques. The controller ensured asymptotic end-effector tracking despite the presence of uncertainties in the robot manipulator dynamics. Experiments performed on a robot manipulator illustrated that the end-effector tracking performance was improved at each period of the desired trajectory. A comparison with a task-space PID controller was also presented. Though the experimental studies are performed on a planar robot, as the theory proposed is not limited to planar robots, it is the authors' sincere belief that, similar performance can also be achieved with a robot manipulator working in 3D. Designing an output feedback version of the proposed task-space controller considered as a possible future research.

\section{REFERENCES}

1. Lewis, F. L., C. T. Abdallah, and D. M. Dawson, Control of Robot Manipulators, MacMillan, New, York (1993).

2. Dawson, D. M., M. M. Bridges, and Z. Qu, Nonlinear Control of Robotic Systems for Environmental Waste and Restoration M. J. U. of New Mexico (Ed.), Vol. 5, Englewood Cliffs, NJ (1995).

3. Arimoto, S., Control Theory of Non-Linear Mechanical Systems: A Passivity-Based and Circuit-Theoretic Approach, Clarendon, Oxford (1996). 
4. Lewis, F. L., D. M. Dawson, and C. T. Abdallah, Robot Manipulator Control: Theory and Practice, Marcel Dekker, New York (2003).

5. Dixon, W. E., A. Behal, D. M. Dawson, and S. P. Nagarkatti, Nonlinear Control of Engineering Systems: A Lyapunov-Based Approach, Birkhauser, Basel, Switzerland (2003).

6. Ioannou, P. and J. Sun, Robust Adaptive Control, Prentice-Hall, Englewood Cliffs, NJ, USA (1996).

7. Lavretsky, E. and K. Wise, Robust and Adaptive Control With Aerospace Applications, Springer, New York (2013).

8. Qu, Z., Robust Control of Nonlinear Uncertain Systems, Wiley, New York (1998).

9. Qu, Z. and J.-X. Xu, "Model-based learning controls and their comparisons using Lyapunov direct method," Asian J. Control, Vol. 4, No. 1, pp. 99-110 (2002).

10. Arimoto, S., S. Kawamura, and F. Miyazaki, "Bettering operation of robots by learning," J. Robot. Syst., Vol. 1, No. 2, pp. 123-140 (1984).

11. Arimoto, S., S. Kawamura, and F. Miyazaki, "Realization of robot motion based on a learning method," IEEE Trans. Syst. Man Cybern., Vol. 18, No. 1, pp. 126-134 (1988).

12. Messner, W., R. Horowitz, W. Kao, and M. Boals, "A new adaptive learning rule," IEEE Trans. Autom. Control, Vol. 36, pp. 188-197 (1991).

13. Horowitz, R., "Learning control of robot manipulators," ASME J. Dynam. Syst. Meas. Control, Vol. 115, pp. 402-411 (1993).

14. Dixon, W. E., E. Zergeroglu, D. M. Dawson, and B. T. Costic, "Repetitive learning control : A Lyapunov-based approach," IEEE Trans. Syst. Man Cybern., Vol. 32, No. 4, pp. 538-545 (2002).

15. Tayebi, A., "Adaptive iterative learning control of robot manipulators," Automatica, Vol. 40, pp. 1195-1203 (2004).

16. Liuzzo, S. and P. Tomei, "A global adaptive learning control of robot manipulators," Automatica, Vol. 44, pp. 1378-1384 (1989).

17. Heinzinger, D., B. Fenwick, B. Paden, and F. Miyazaki, "Robust learning controll," Proc. IEEE Int. Conf. Decis. Control, Tampa, FL, USA, pp. 436-440 (1989).

18. Tian, H. and Y. Su, "A repetitive learning method based on sliding mode for robot control with actuator saturation," J. Dyn. Syst. Meas. Control, Vol. 137, pp. 064 505-1 (2015).

19. Marino, R., P. Tomei, and C. Verrelli, "Learning control for nonlinear systems in output feedback form," Syst. Control Lett., Vol. 61, pp. 1242-1247 (2012).
20. Scalzi, S., S. Bifaretti, and C. M. Verrelli, "Repetitive learning control design for LED light tracking," IEEE Trans Control Syst. Technol., Vol. 23, No. 3, pp. 1139-1146 (2015).

21. Verrelli, C. M., "Repetitive learning control design and period uncertainties," Asian J. Control, Vol. 17, No. 6, pp. 2417-2426 (2015).

22. Verrelli, C. M., "A larger family of nonlinear systems for the repetitive learning control," Automatica, Vol. 71, pp. 38-43 (2016).

23. Verrelli, C. M., P. Tomei, V. Salis, and S. Bifaretti, "Repetitive learning position control for full order model permanent magnet step motors," Automatica, Vol. 63, pp. 274-286 (2016).

24. Sekimoto, M., S. Arimoto, S. Umesao, T. Torii, and H. Hahiguchi, "Iterative learning control in task-space for robots with redundant joints," $J$. Robot. Syst., Vol. 25, No. 6, pp. 921-929 (2007).

25. Arimoto, S., M. Sekimoto, and S. Kawamura, "Task-space iterative learning for redundant robotic systems: Existence of a task-space control and convergence of learning," SICE J. Control Meas. Syst. Integr., Vol. 1, No. 4, pp. 312-319 (2008).

26. Inverse kinematic problem solving: A symbolic approach using maplesim and maple. [Online]. Available: www.maplesoft.com.

27. Dogan, K. M., E. Tatlicioglu, and E. Zergeroglu, "Operational/task space learning control of robot manipulators with dynamical uncertainties," Proc. IEEE Int. Conf. Control Applicat., Sydney, Australia, pp. 527-532 (2015).

28. Nakanishi, J., R. Cory, M. M. J. Peters, and S. Schaal, "Operational space control: A theoretical and emprical comparsion," Int. J. Robot. Res., Vol. 27, No. 6, pp. 737-757 (2008).

29. Sadegh, N. and R. Horowitz, "Stability and robustness analysis of a class of adaptive controllers for robot manipulators," Int. J. Robot. Res., Vol. 9, No. 3, pp. 74-92 (1990).

30. Dasdemir, J. and E. Zergeroglu, "A new continuous high-gain controller scheme for a class of uncertain nonlinear systems," Int. J. Robust Nonlinear Control, Vol. 25, No. 1, pp. 125-141 (2015).

31. Costic, B., M. S. deQueiroz, and D. M. Dawson, "A new learning control approach to the active magnetic bearing benchmark system," Proc. Amer. Control Conf., Chicago, IL, pp. 5989-5994 (2000).

32. Khalil, H., Nonlinear Systems, 3rd Edition, Prentice Hall, Englewood Cliffs, NJ, USA (2002). 


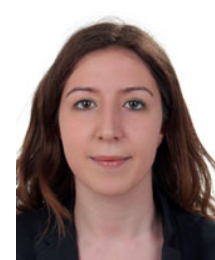

K. Merve Dogan is a graduate student of the Department of Mechanical Engineering at the University of South Florida. She received the B.Sc. degree from Pamukkale University in 2012 and the M.Sc. degree from Izmir Institute of Technology in 2016. Her dynamical systems and controls research specializes in adaptive control, robust control, and convex optimization with applications to robotics, human-machine interactions, and autonomous vehicles.

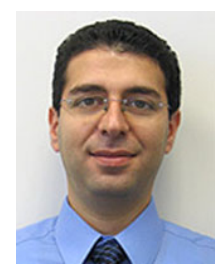

Enver Tatlicioglu received the B.Sc. degree in Electrical \& Electronics Engineering from Dokuz Eylul University, Izmir, Turkey and the Ph.D. degree in Electrical and Computer Engineering from Clemson University, Clemson, SC, USA in 1999 and 2007, respectively. Upon completion of his Ph.D. degree, he worked as a post-doctoral research fellow in the Department of Electrical and Computer Engineering at Clemson University then he joined the Department of Electrical \& Electronics Engineering at Izmir Institute of Technology, Izmir, Turkey where he is currently a full professor. His research interests include control and identification of time delay systems, dynamic modelling of extensible continuum robot manipulators, non-linear control techniques for kinematically redundant robot manipulators, partial state feedback and output feedback control, haptic systems and teleoperation; learning, robust and adaptive control of non-linear systems.

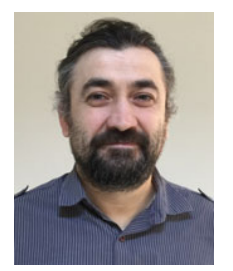

Erkan Zergeroglu received his B.Sc. and M.Sc. degrees in Electrical and Electronic Engineering from Hacettepe University and Middle East Technical University in 1992 and 1996, respectively, both in Turkey. In 2000 he received his Ph.D. degree in Electrical Engineering from Clemson University, South Carolina, USA. He then joined Lucent Technologies-Bell Labs Innovations, Optical Fiber Solutions, Sturbridge MA as Member of Technical Stuff from 2000-2002. In 2002, he joined his Computer Engineering Department of Gebze Institute of Technology (which in 2015 renamed as Gebze Technical University) Kocaeli-Turkey, where he currently leads the Control Technologies and Robotics Laboratory. His main research interests are in the fields of nonlinear model-based robust, adaptive, and learning control with application to electro-mechanical systems, including but not limited to robotics and control of robot manipulators, visual servoing applications, and variable speed wind turbines.

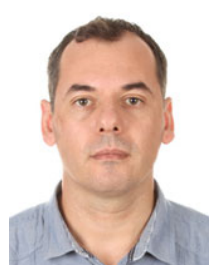

Kamil Cetin received the B.Sc. degree in Electrical and Electronics Engineering from Dumlupinar University, Turkey in June 2002, the M.Sc. degree in Electrical and Electronics Engineering from Dokuz Eylul University, Turkey in April 2007, and the Ph.D. degree in Electronics and Communication Engineering from Izmir Institute of Technology, Turkey in October 2016. He worked as an electronic engineer in an industrial company in Izmir, Turkey between 2004 and 2008, he pursued his career as a research assistant in the Electronic Systems Department at Aalborg University, Aalborg, Denmark between 2008 and 2011. During his doctoral studies, he worked within a national project supported by The Scientific and Technological Research Council of Turkey. He is working as a post-doctoral researcher in the Robotics Brain Cognitive and Science Department at the Italian Institute of Technology since May 2017. His research interests include robot manipulators, telerobotic systems, nonlinear control methods, and cable-driven robots. 